\title{
Elasticity theory of smectic and canonic mesophases
}

\author{
S. Stallinga and G. Vertogen \\ Institute for Theoretical Physics, University of Nijmegen, Toernooiveld, 6525 ED Nijmegen, The Netherlands
}

(Received 7 January 1994; revised manuscript received 18 July 1994)

\begin{abstract}
The general theory of elasticity for smectic and canonic mesophases is formulated, starting from the assumption that the equilibrium state is spatially periodic. The various surface terms appearing in the deformation free energy density are considered as well. The effective description of the elastic behavior of a general nonchiral smectic mesophase involves one positional elastic constant, 16 bulk orientational elastic constants, and six surface orientational elastic constants. One additional bulk orientational elastic constant is required for the description of a general chiral smectic mesophase. The effective description of the elastic behavior of a general nonchiral canonic mesophase involves six positional elastic constants and three bulk orientational elastic constants. In this case the property of chirality does not introduce additional orientational elastic constants. The elastic constants for some relevant smectic and canonic mesophases are given, including the elastic constants for the antiferroelectric $\mathrm{Sm}-C_{A}^{*}$ and ferrielectric $\mathrm{Sm}-C_{\alpha}^{*}$ and $\mathrm{Sm}-C_{\gamma}^{*}$ phases.
\end{abstract}

PACS number(s): 61.30. $-\mathrm{v}, 03.40 . \mathrm{Dz}$

\section{INTRODUCTION}

The present work deals with the description of the elastic behavior of smectic and canonic mesophases, or shortly smectics and canonics. Smectics are solid in one dimension and fluid in the other two dimensions, i.e., they have a broken continuous translational symmetry in one direction. The only possible axis of continuous rotational symmetry coincides with this preferential direction. Canonics, on the other hand, are solids in two dimensions and fluid in the other dimension, i.e., they have a broken translational symmetry in two independent directions. Here, axes of continuous rotational symmetry are absent.

The elasticity theory of smectic and canonic mesophases must take into account the interdependence of the positional and orientational deformation variables. The breaking of continuous translational symmetry is described in terms of a displacement field $u(r)$, which describes the deviation of a material point situated at $r$ from its equilibrium position $\boldsymbol{r}-\boldsymbol{u}(\boldsymbol{r})$. The breaking of continuous rotational symmetry is described in terms of an orientational field consisting of three orthonormal vectors $\boldsymbol{a}(\boldsymbol{r}), \boldsymbol{b}(\boldsymbol{r})$, and $\boldsymbol{c}(\boldsymbol{r})$, which are attached to each material point. Equivalently, the local orientation is described by the orthogonal transformation matrix, that connects the local body-fixed frame and the space-fixed frame. The matrix elements $R_{i \alpha}$ with $i=1,2,3$ and $\alpha=x, y, z$ are given by

$$
\begin{aligned}
& R_{1 \alpha}=b_{\alpha}, \\
& R_{2 \alpha}=c_{\alpha}, \\
& R_{3 \alpha}=a_{\alpha} .
\end{aligned}
$$

The interdependence of the positional and orientational deformation variables is formulated in terms of constraints on these variables. These constraints express mathematically the nature of the smectic and canonic mesophases.

The theory of elasticity is concerned with expressions for the deformation free energy density, i.e., the excess free energy density of a deformed state with respect to the state with uniform displacement and orientational field. This deformation free energy density is a function of the spatial derivatives of the deformation variables. In smectics and canonics positional as well as orientational elasticity must be taken into account. For the sake of clearness the essential features of the theories of both positional and orientational elasticity are summarized. A detailed exposition of these theories can be found in references [1] and [2], respectively.

The general expression of the free energy density due to positional elasticity is given by

$$
f_{p}=\frac{1}{2} \lambda_{\alpha \beta \gamma \delta} U_{\alpha \beta} U_{\gamma \delta}
$$

where $\lambda_{\alpha \beta \gamma \delta}=\lambda_{\gamma \delta \alpha \beta}$ is the elasticity tensor and where the strain tensor $U_{\alpha \beta}$ is defined by

$$
U_{\alpha \beta}=\frac{1}{2}\left(\partial_{\alpha} u_{\beta}+\partial_{\beta} u_{\alpha}-\partial_{\alpha} u_{\gamma} \partial_{\beta} u_{\gamma}\right)
$$

Terms linear in $U_{\alpha \beta}$ do not appear, as the undeformed state is assumed to be the equilibrium state. Terms of higher than second order in $U_{\alpha \beta}$ are neglected, as the strain tensor is assumed to be small. The symmetry of the strain tensor leads to the symmetry relations:

$$
\lambda_{\alpha \beta \gamma \delta}=\lambda_{\beta \alpha \gamma \delta}=\lambda_{\alpha \beta \delta \gamma} .
$$

The positional part of the deformation free energy density $f_{p}$ does not contain surface terms, i.e., terms that can be written as divergences and consequently only contribute to the surface free energy. The proof is given in the Appendix.

The elasticity tensor can be expressed as 


$$
\lambda_{\alpha \beta \gamma \delta}=R_{i \alpha} R_{j \beta} R_{k \gamma} R_{l \delta} \lambda_{i j k l},
$$

where the scalars $\lambda_{i j k l}$ are the elastic constants. It follows directly that the elastic constants must satisfy the symmetry relations:

$$
\lambda_{i j k l}=\lambda_{j i k l}=\lambda_{i j l k}=\lambda_{k l i j} .
$$

The general expression of the positional deformation free energy density involves 21 independent elastic constants.

As to the deformation free energy density due to orientational elasticity, the general expression is given by

$$
f_{o}=k_{i j} D_{i j}+\frac{1}{2} K_{i j k l} D_{i j} D_{k l}+L_{i j k} S_{i j k}
$$

where

$$
\begin{aligned}
D_{i j} & =\frac{1}{2} \varepsilon_{j k l} R_{i \alpha} R_{k \beta} \partial_{\alpha} R_{l \beta}, \\
S_{i j k}=S_{j i k} & =\partial_{\alpha}\left(R_{i \alpha} D_{j k}+R_{j \alpha} D_{i k}\right),
\end{aligned}
$$

and $k_{i j}, K_{i j k l}=K_{k l i j}$ and $L_{i j k}=L_{j i k}$ are the elastic constants. It appears that there are 9 elastic constants $k_{i j}, 45$ elastic constants $K_{i j k l}$, and 18 elastic constants $L_{i j k}$. The invariants $D_{i j}$ can be written in terms of the usual vector notation as

$$
\begin{aligned}
& D_{11}=\frac{1}{2}[\boldsymbol{c} \cdot(\boldsymbol{\nabla} \times \boldsymbol{c})+\boldsymbol{a} \cdot(\boldsymbol{\nabla} \times \boldsymbol{a})-\boldsymbol{b} \cdot(\boldsymbol{\nabla} \times \boldsymbol{b})], \\
& D_{12}=-\boldsymbol{c} \cdot(\boldsymbol{\nabla} \times \boldsymbol{b}), \\
& D_{13}=-\boldsymbol{a} \cdot(\boldsymbol{\nabla} \times \boldsymbol{b}), \\
& D_{21}=-\boldsymbol{b} \cdot(\boldsymbol{\nabla} \times \boldsymbol{c}), \\
& D_{22}=\frac{1}{2}[\boldsymbol{a} \cdot(\boldsymbol{\nabla} \times \boldsymbol{a})+\boldsymbol{b} \cdot(\boldsymbol{\nabla} \times \boldsymbol{b})-\boldsymbol{c} \cdot(\boldsymbol{\nabla} \times \boldsymbol{c})], \\
& D_{23}=-\boldsymbol{a} \cdot(\boldsymbol{\nabla} \times \boldsymbol{c}), \\
& D_{31}=-\boldsymbol{b} \cdot(\boldsymbol{\nabla} \times \boldsymbol{a}), \\
& D_{32}=-\boldsymbol{c} \cdot(\boldsymbol{\nabla} \times \boldsymbol{a}), \\
& D_{33}=\frac{1}{2}[\boldsymbol{b} \cdot(\boldsymbol{\nabla} \times \boldsymbol{b})+\boldsymbol{c} \cdot(\boldsymbol{\nabla} \times \boldsymbol{c})-\boldsymbol{a} \cdot(\boldsymbol{\nabla} \times \boldsymbol{a})] .
\end{aligned}
$$

As opposed to the case of translational elasticity the orientational deformation free energy density does involve surface terms. These surface terms are the 18 terms $S_{i j k}$, the three chiral terms,

$$
S_{i}=\partial_{\alpha} R_{i \alpha}=-\varepsilon_{i j k} D_{j k},
$$

and the six nonchiral terms,

$$
\begin{aligned}
S_{i j} & =\partial_{\alpha}\left(R_{i \beta} \partial_{\beta} R_{j \alpha}-R_{i \alpha} \partial_{\beta} R_{j \beta}\right) \\
& =\left(\varepsilon_{k i m} \varepsilon_{l j n}-\varepsilon_{l i m} \varepsilon_{k j n}\right) D_{l m} D_{k n}
\end{aligned}
$$

It can be shown that the surface terms $S_{i}, S_{i j}$, and $S_{i j k}$ are all possible surface terms [2]. Clearly, a further reduction of the number of bulk terms is not possible.

Mixing terms proportional to $D_{i j} U_{\alpha \beta}$ do not appear in $f_{d}$, as the state with uniform displacement field is assumed to be the equilibrium state. Consequently, the deformation free energy density is the sum of the positional and the orientational contribution:

$$
f_{d}=f_{p}+f_{o} .
$$

The expression (12) is the starting point of the elastic- ity theory of smectic and canonic mesophases. The explicit expressions, however, must take into account the macroscopic definition of these mesophases in terms of the interdependence of the positional and orientational variables.

The paper is organized in the following way. In section II, the interdependence of the deformation variables of smectics is formulated and expressions for $f_{p}$ and $f_{o}$ are given. In Sec. III, an analogous procedure is followed for canonics. Finally, the results are discussed in Sec. IV.

\section{ELASTICITY THEORY OF SMECTIC MESOPHASES}

\section{A. Deformations in smectics}

The orientational field in a smectic is chosen such that the vector $\boldsymbol{a}(\boldsymbol{r})$ is normal to the fluid layers, which consequently are spanned by the vectors $\boldsymbol{b}(\boldsymbol{r})$ and $\boldsymbol{c}(\boldsymbol{r})$. Smectic mesophases are characterized macroscopically by two properties.

The first property is the broken continuous translational symmetry in the direction of the vector $\boldsymbol{a}(\boldsymbol{r})$, whereas the continuous translational symmetry is unbroken in the surface perpendicular to $\boldsymbol{a}(\boldsymbol{r})$. The starting point of the present analysis is the assumption that the equilibrium state is the state with spatial periodicity, i.e., the undistorted smectic layers are flat. Then the distorted layer is described by the requirement that the function $\Phi(\boldsymbol{r})=\boldsymbol{k} \cdot[\boldsymbol{r}-\boldsymbol{u}(\boldsymbol{r})]$, where $\boldsymbol{k}$ is perpendicular to the undeformed planes, must equal a constant. The function $\Phi(\boldsymbol{r})$ is the so-called layer phase function [3]. Choosing $\boldsymbol{k}=\hat{\boldsymbol{e}}_{\boldsymbol{z}}$ it, follows that

$$
\Phi(\boldsymbol{r})=z-u_{z}(\boldsymbol{r}) .
$$

Clearly $u_{x}(\boldsymbol{r})$ and $u_{y}(\boldsymbol{r})$ do not enter the theory.

The second property relates the orientational variables to the positional variable $u_{z}(\boldsymbol{r})$. As the unit vector $\boldsymbol{a}(\boldsymbol{r})$ is perpendicular to the fluid layers this second property can be expressed mathematically as

$$
\begin{aligned}
\boldsymbol{a}(\boldsymbol{r}) & =\frac{\boldsymbol{\nabla} \Phi(\boldsymbol{r})}{|\boldsymbol{\nabla} \Phi(\boldsymbol{r})|} \\
& =\frac{\left(-\partial_{x} u_{z},-\partial_{y} u_{z}, 1-\partial_{z} u_{z}\right)}{\sqrt{\left(\partial_{x} u_{z}\right)^{2}+\left(\partial_{y} u_{z}\right)^{2}+\left(1-\partial_{z} u_{z}\right)^{2}}} .
\end{aligned}
$$

Using the representation in Eulerian angles:

$$
\begin{aligned}
\boldsymbol{a}= & (\sin \theta \cos \phi, \sin \theta \sin \phi, \cos \theta), \\
\boldsymbol{b}= & \cos (\psi)(\cos \theta \cos \phi, \cos \theta \sin \phi,-\sin \theta) \\
& +\sin (\psi)(-\sin \phi, \cos \phi, 0), \\
\boldsymbol{c}= & -\sin (\psi)(\cos \theta \cos \phi, \cos \theta \sin \phi,-\sin \theta) \\
& +\cos (\psi)(-\sin \phi, \cos \phi, 0),
\end{aligned}
$$

it follows directly that

$$
\begin{aligned}
& \cos \theta=\frac{1-\partial_{z} u_{z}}{|\nabla \Phi|}, \\
& \sin \theta=\frac{\left|\nabla_{\perp} \Phi\right|}{|\nabla \Phi|},
\end{aligned}
$$


and

$$
\begin{aligned}
& \cos \phi=-\frac{\partial_{x} u_{z}}{\left|\nabla_{\perp} \Phi\right|}, \\
& \sin \phi=-\frac{\partial_{y} u_{z}}{\left|\nabla_{\perp} \Phi\right|}
\end{aligned}
$$

where

$$
\nabla_{\perp} \Phi=\left(-\partial_{x} u_{z},-\partial_{y} u_{z}, 0\right)
$$

Expressions for the axes $\boldsymbol{b}$ and $\boldsymbol{c}$ can now be easily found:

$$
\begin{aligned}
\boldsymbol{b}= & \frac{\cos \psi}{|\nabla \Phi|\left|\nabla_{\perp} \Phi\right|}\left(-\partial_{x} u_{z}\left(1-\partial_{z} u_{z}\right),-\partial_{y} u_{z}\left(1-\partial_{z} u_{z}\right)\right. \\
& \left.-\left(\partial_{x} u_{z}\right)^{2}-\left(\partial_{y} u_{z}\right)^{2}\right) \\
& +\frac{\sin \psi}{\left|\nabla_{\perp} \Phi\right|}\left(\partial_{y} u_{z},-\partial_{x} u_{z}, 0\right), \\
\boldsymbol{c}= & \frac{-\sin \psi}{|\nabla \Phi|\left|\nabla_{\perp} \Phi\right|}\left(-\partial_{x} u_{z}\left(1-\partial_{z} u_{z}\right),-\partial_{y} u_{z}\left(1-\partial_{z} u_{z}\right)\right. \\
& \left.-\left(\partial_{x} u_{z}\right)^{2}-\left(\partial_{y} u_{z}\right)^{2}\right) \\
& +\frac{\cos \psi}{\left|\nabla_{\perp} \Phi\right|}\left(\partial_{y} u_{z},-\partial_{x} u_{z}, 0\right) .
\end{aligned}
$$

The Eulerian angle $\psi$ corresponds to the only independent angular variable, describing the rotation around the local normal $\boldsymbol{a}$. Clearly, the orientational field is a function of $\partial_{\alpha} u_{z}$ and $\psi$.

\section{B. Positional elasticity}

The strain tensor can be expressed as

$$
U_{\alpha \beta}=\frac{1}{2}\left[\delta_{\alpha \beta}-\partial_{\alpha} \Phi_{\gamma} \partial_{\beta} \Phi_{\gamma}\right]
$$

where $\boldsymbol{\Phi}(\boldsymbol{r})=\boldsymbol{r}-\boldsymbol{u}(\boldsymbol{r})$ gives the position of a material point in the undeformed state as a function of its position in the deformed state. The nature of the smectic state imposes a constraint on the general form of the strain tensor (20). Because of the fluid character of the smectic layers only the component of $\boldsymbol{\Phi}$ perpendicular to the undistorted layers is relevant for the strain, i.e., appears in the strain tensor. Both other components do not contribute. Using (14), i.e.,

$$
\partial_{\alpha} \Phi_{z}=\partial_{\alpha} \Phi=|\nabla \Phi| a_{\alpha}
$$

the strain tensor of a smectic is found to be

$$
\begin{aligned}
U_{\alpha \beta} & =\frac{1}{2}\left[\delta_{\alpha \beta}-|\nabla \Phi|^{2} a_{\alpha} a_{\beta}\right] \\
& =\frac{1}{2}\left(b_{\alpha} b_{\beta}+c_{\alpha} c_{\beta}\right)+\varepsilon a_{\alpha} a_{\beta},
\end{aligned}
$$

where $\varepsilon$ is given by

$$
\begin{aligned}
\varepsilon & =\frac{1}{2}\left(1-|\nabla \Phi|^{2}\right) \\
& =\partial_{z} u_{z}-\frac{1}{2}\left\{\left(\partial_{x} u_{z}\right)^{2}+\left(\partial_{y} u_{z}\right)^{2}+\left(\partial_{z} u_{z}\right)^{2}\right\} .
\end{aligned}
$$

Substitution of this expression for the strain tensor in the general formula (2) for the positional deformation free energy density gives

$$
\begin{aligned}
f_{p}= & \frac{1}{2} \lambda_{3333} \varepsilon^{2}+\frac{1}{2}\left(\lambda_{1133}+\lambda_{2233}\right) \varepsilon \\
& +\frac{1}{8}\left(\lambda_{1111}+\lambda_{2222}+2 \lambda_{1122}\right) .
\end{aligned}
$$

The term linear in $\varepsilon$ is forbidden for reasons of stability. Leaving out the irrelevant constant term and defining

$$
\lambda=\lambda_{3333},
$$

the positional deformation free energy density of a smectic appears to read,

$$
\begin{aligned}
f_{p} & =\frac{1}{2} \lambda\left(a_{\alpha} a_{\beta} U_{\alpha \beta}\right)^{2} \\
& =\frac{1}{2} \lambda \varepsilon^{2} \\
& =\frac{1}{2} \lambda\left(\partial_{z} u_{z}-\frac{1}{2}\left\{\left(\partial_{x} u_{z}\right)^{2}+\left(\partial_{y} u_{z}\right)^{2}+\left(\partial_{z} u_{z}\right)^{2}\right\}\right)^{2} .
\end{aligned}
$$

This term which is the only contribution to positional elasticity in smectics can be ascribed to the compression of the smectic layers. It should be noted that it is imperative to use the full strain tensor, $U_{\alpha \beta}$ instead of the usual linearized strain tensor,

$$
U_{\alpha \beta}^{\operatorname{lin}}=\frac{1}{2}\left(\partial_{\alpha} u_{\beta}+\partial_{\beta} u_{\alpha}\right)
$$

in order to ensure that the positional deformation free energy density is invariant with respect to rigid rotations of the smectic. See for a further discussion of this point Ref. [4] on the elasticity of Sm- $A$.

\section{Orientational elasticity}

Expressions for the orientational invariants $D_{i j}$ in terms of the spatial derivatives of $\partial_{\alpha} u_{z}$ and $\psi$ can be derived using equations (14) and (19). The spatial derivative of the local normal can be expressed as

$$
\begin{aligned}
\partial_{\alpha} a_{\beta} & =\partial_{\alpha}\left[\frac{\partial_{\beta} \Phi}{|\nabla \Phi|}\right] \\
& =\frac{\partial_{\alpha} \partial_{\beta} \Phi}{|\nabla \Phi|}-\frac{\partial_{\beta} \Phi \partial_{\nu} \Phi \partial_{\alpha} \partial_{\nu} \Phi}{|\nabla \Phi|^{3}} \\
& =\frac{\left(\delta_{\beta \nu}-a_{\beta} a_{\nu}\right) \partial_{\alpha} \partial_{\nu} \Phi}{|\nabla \Phi|} \\
& =-\frac{b_{\beta} b_{\nu}+c_{\beta} c_{\nu}}{|\nabla \Phi|} \partial_{\alpha} \partial_{\nu} u_{z} .
\end{aligned}
$$

Six invariants $D_{i j}$ follow now directly:

$$
D_{11}=b_{\alpha} c_{\beta} \partial_{\alpha} a_{\beta}=-\frac{1}{|\nabla \Phi|} b_{\alpha} c_{\beta} \partial_{\alpha} \partial_{\beta} u_{z}
$$




$$
\begin{aligned}
& D_{21}=c_{\alpha} c_{\beta} \partial_{\alpha} a_{\beta}=-\frac{1}{|\nabla \Phi|} c_{\alpha} c_{\beta} \partial_{\alpha} \partial_{\beta} u_{z} \\
& D_{31}=a_{\alpha} c_{\beta} \partial_{\alpha} a_{\beta}=-\frac{1}{|\nabla \Phi|} a_{\alpha} c_{\beta} \partial_{\alpha} \partial_{\beta} u_{z} \\
& D_{12}=-b_{\alpha} b_{\beta} \partial_{\alpha} a_{\beta}=\frac{1}{|\nabla \Phi|} b_{\alpha} b_{\beta} \partial_{\alpha} \partial_{\beta} u_{z} \\
& D_{22}=-c_{\alpha} b_{\beta} \partial_{\alpha} a_{\beta}=\frac{1}{|\nabla \Phi|} c_{\alpha} b_{\beta} \partial_{\alpha} \partial_{\beta} u_{z} \\
& D_{32}=-a_{\alpha} b_{\beta} \partial_{\alpha} a_{\beta}=\frac{1}{|\nabla \Phi|} a_{\alpha} b_{\beta} \partial_{\alpha} \partial_{\beta} u_{z}
\end{aligned}
$$

In order to obtain the three remaining invariants the vector $b_{\beta} \partial_{\alpha} c_{\beta}$ is expressed in terms of Eulerian angles:

$$
b_{\beta} \partial_{\alpha} c_{\beta}=-\cos (\theta) \partial_{\alpha} \phi-\partial_{\alpha} \psi
$$

Next $\partial_{\alpha} \phi$ is expressed in terms of the derivatives of the displacement field. Consider to that end:

$$
\tan \phi=\frac{\partial_{y} u_{z}}{\partial_{x} u_{z}} .
$$

Then it follows that

$$
\begin{aligned}
\partial_{\alpha} \phi & =\frac{1}{1+\tan ^{2} \phi} \partial_{\alpha}(\tan \phi) \\
& =\frac{1}{\left|\nabla_{\perp} \Phi\right|^{2}}\left(\partial_{x} u_{z} \partial_{\alpha} \partial_{y} u_{z}-\partial_{y} u_{z} \partial_{\alpha} \partial_{x} u_{z}\right) \\
& =-\frac{1}{\left|\nabla_{\perp} \Phi\right|}\left[\sin (\psi) b_{\beta}+\cos (\psi) c_{\beta}\right] \partial_{\alpha} \partial_{\beta} u_{z}
\end{aligned}
$$

Consequently, the remaining three invariants $D_{i j}$ can be written as

$$
\begin{aligned}
& D_{13}=b_{\alpha} b_{\beta} \partial_{\alpha} c_{\beta} \\
& =\frac{1-\partial_{z} u_{z}}{|\nabla \Phi|\left|\nabla_{\perp} \Phi\right|}\left[\cos (\psi) b_{\alpha} c_{\beta} \partial_{\alpha} \partial_{\beta} u_{z}\right. \\
& \left.+\sin (\psi) b_{\alpha} b_{\beta} \partial_{\alpha} \partial_{\beta} u_{z}\right]-b_{\alpha} \partial_{\alpha} \psi \\
& D_{23}=c_{\alpha} b_{\beta} \partial_{\alpha} c_{\beta} \\
& =\frac{1-\partial_{z} u_{z}}{|\nabla \Phi|\left|\nabla_{\perp} \Phi\right|}\left[\cos (\psi) c_{\alpha} c_{\beta} \partial_{\alpha} \partial_{\beta} u_{z}\right. \\
& \left.+\sin (\psi) c_{\alpha} b_{\beta} \partial_{\alpha} \partial_{\beta} u_{z}\right]-c_{\alpha} \partial_{\alpha} \psi, \\
& D_{33}=a_{\alpha} b_{\beta} \partial_{\alpha} c_{\beta} \\
& =\frac{1-\partial_{z} u_{z}}{|\boldsymbol{\nabla} \Phi|\left|\nabla_{\perp} \Phi\right|}\left[\cos (\psi) a_{\alpha} c_{\beta} \partial_{\alpha} \partial_{\beta} u_{z}\right. \\
& \left.+\sin (\psi) a_{\alpha} b_{\beta} \partial_{\alpha} \partial_{\beta} u_{z}\right]-a_{\alpha} \partial_{\alpha} \psi \text {. }
\end{aligned}
$$

It follows immediately from (29) that

$$
\boldsymbol{a} \cdot(\boldsymbol{\nabla} \times \boldsymbol{a})=D_{11}+D_{22}=0 .
$$

This relation can also be derived more directly with the aid of Eq. (14) [3].

The specific character of a smectic thus reduces the number of rotational invariants from nine to eight. From an experimental point of view, however, a description in terms of six invariants suffices, as the influence of the two invariants $D_{31}$ and $D_{32}$ can be neglected. This can be seen as follows. Both invariants only appear in case of a deformation that involves layer compression. Consider such a deformation with wave vector of magnitude $q$, where the wavelength of the distortion is much larger than a molecular length as required by continuum theory. The contribution to the deformation free energy density due to positional elasticity is then of order $\lambda q^{2}$, whereas the contribution due to orientational elasticity is of order $K q^{4}$, as follows from the proportionality of the invariants $D_{i j}$ to the second order derivatives of the displacement field. Empirically, it appears that the length $\sqrt{K / \lambda}$ is of the order of a molecular length, as also follows from considerations concerning the molecular constituency of smectics. Consequently, the ratio $K q^{2} / \lambda$ of the two contributions is much smaller than one, i.e., the influence of the invariants $D_{31}$ and $D_{32}$ can be neglected.

The invariants $D_{31}$ and $D_{32}$ vanish automatically as soon as the smectic layers are required to be incompressible [3]. This requirement can be put in mathematical terms in the following way:

$$
|\nabla \Phi|=1 \text {. }
$$

Then, it holds according to Eq. (14) that

$$
\boldsymbol{\nabla} \times \boldsymbol{a}=\mathbf{0} .
$$

This irrotationality condition was introduced for the first time by Oseen [5]. It entails the exact result (34) and

$$
\begin{aligned}
& D_{31}=-\boldsymbol{b} \cdot(\boldsymbol{\nabla} \times \boldsymbol{a})=0, \\
& D_{32}=-\boldsymbol{c} \cdot(\boldsymbol{\nabla} \times \boldsymbol{a})=0 .
\end{aligned}
$$

Equivalently, it can be said that the elastic constants associated with the three invariants $D_{11}+D_{22}, D_{31}$, and $D_{32}$ are infinite.

Summarizing, the deformations of the orientational field in smectics can be effectively described by six invariants. Consequently, 21 elastic constants $K_{i j k l}$ appear in the orientational deformation free energy density for general smectics, satisfying the irrotationality constraint. This requirement of incompressibility also reduces the number of surface terms $S_{i j}$, given in equation (11), from six to five. Thus, 16 elastic constants suffice for a general description of the bulk of general incompressible smectics.

The number of chiral terms is further limited by the assumption that the equilibrium state is the state with spatial periodicity. This means that terms linear in the derivatives of the displacement field do not appear in the deformation free energy density, i.e., chiral terms proportional to the the second order derivatives of the displacement field are not allowed to be present. Consequently, only the chiral elastic constant $k_{33}$ appears, as the attendant term does not contain second order derivatives of the displacement field. As to the remaining surface terms $S_{i j k}$, only the term $S_{333}$ is relevant for the description of smectic elasticity, for the other terms are forbidden by the requirement of incompressibility or by the assumption concerning the nature of the equilibrium state. Finally, it should be realized that the correctness of the assumption concerning the equilibrium state must be decided experimentally. 


\section{ELASTICITY THEORY OF CANONIC MESOPHASES}

\section{A. Deformations in canonics}

The orientational field in a canonic is chosen such that the solid layers are spanned by the two vectors $\boldsymbol{b}(\boldsymbol{r})$ and $\boldsymbol{c}(\boldsymbol{r})$. Consequently, the vector $\boldsymbol{a}(\boldsymbol{r})$ is directed along the fluid columns. Canonic mesophases are also characterized macroscopically by two properties.

The first property is the broken continuous translational symmetry in the surface perpendicular to $\boldsymbol{a}(\boldsymbol{r})$, whereas the continuous translational symmetry is unbroken in the direction along $\boldsymbol{a}(\boldsymbol{r})$. Starting point of the analysis is also here the assumption that the equilibrium state is the state with spatial periodicity, i.e., the undistorted canonic columns are straight. Then the distorted column is described by the requirement that the functions $\Phi_{1}(r)=k_{1} \cdot[r-u(r)]$ and $\Phi_{2}(r)=k_{2} \cdot[r-u(r)]$, where $\boldsymbol{k}_{1}$ and $\boldsymbol{k}_{2}$ are two linearly independent vectors perpendicular to the undeformed columns, must equal a constant. The functions $\Phi_{1}(\boldsymbol{r})$ and $\Phi_{2}(\boldsymbol{r})$ are the generalization of the layer phase function for smectics. Choosing $\boldsymbol{k}_{1}=\hat{\boldsymbol{e}}_{\boldsymbol{x}}$ and $\boldsymbol{k}_{2}=\hat{\boldsymbol{e}}_{\boldsymbol{y}}$, it follows that

$$
\begin{aligned}
& \Phi_{1}(\boldsymbol{r})=x-u_{x}(\boldsymbol{r}), \\
& \Phi_{2}(\boldsymbol{r})=y-u_{y}(\boldsymbol{r}) .
\end{aligned}
$$

Clearly, $u_{z}(\boldsymbol{r})$ does not enter the theory.

The second property relates the orientational variables to the positional variables $u_{x}(\boldsymbol{r})$ and $u_{y}(\boldsymbol{r})$. As the unit vector $\boldsymbol{a}(\boldsymbol{r})$ is parallel to the fluid columns it is perpendicular to the plane spanned by the two unit vectors

$$
\begin{aligned}
\boldsymbol{b}_{\mathbf{0}} & =\frac{\boldsymbol{\nabla} \Phi_{1}}{\left|\nabla \Phi_{1}\right|} \\
& =\frac{\left(1-\partial_{x} u_{x},-\partial_{y} u_{x},-\partial_{z} u_{x}\right)}{\sqrt{\left(1-\partial_{x} u_{x}\right)^{2}+\left(\partial_{y} u_{x}\right)^{2}+\left(\partial_{z} u_{x}\right)^{2}}}
\end{aligned}
$$

$$
\begin{aligned}
c_{0} & =\frac{\nabla \Phi_{2}}{\left|\nabla \Phi_{2}\right|} \\
& =\frac{\left(-\partial_{x} u_{y}, 1-\partial_{y} u_{y},-\partial_{z} u_{y}\right)}{\sqrt{\left(1-\partial_{y} u_{y}\right)^{2}+\left(\partial_{x} u_{y}\right)^{2}+\left(\partial_{z} u_{y}\right)^{2}}} .
\end{aligned}
$$

Hence, the second canonic property can be mathematically expressed as

$$
\begin{aligned}
a & =\frac{b_{0} \times c_{0}}{\left|b_{0} \times c_{0}\right|} \\
& =\frac{\nabla \Phi_{1} \times \nabla \Phi_{2}}{\sqrt{\left|\nabla \Phi_{1}\right|^{2}\left|\nabla \Phi_{2}\right|^{2}-\left(\nabla \Phi_{1} \cdot \nabla \Phi_{2}\right)^{2}}} .
\end{aligned}
$$

The other two axes can be chosen as

$$
\begin{aligned}
& b=\frac{1}{2} \frac{b_{0}+c_{0}}{\sqrt{1+b_{0} \cdot c_{0}}}+\frac{1}{2} \frac{b_{0}-c_{0}}{\sqrt{1-b_{0} \cdot c_{0}}}, \\
& c=\frac{1}{2} \frac{b_{0}+c_{0}}{\sqrt{1+b_{0} \cdot c_{0}}}-\frac{1}{2} \frac{b_{0}-c_{0}}{\sqrt{1-b_{0} \cdot c_{0}}} .
\end{aligned}
$$

This choice is not unique, as a rotation around $a$ gives an equivalent set of local axes. In contrast to the smectic case an additional angular variable to specify the local orientation is not needed, as the broken continuous rotational symmetry around the $\boldsymbol{a}$ axis is not an independently broken symmetry. Clearly, the orientational field is a function of $\partial_{\alpha} u_{x}$ and $\partial_{\alpha} u_{y}$.

\section{B. Positional elasticity}

The nature of the canonic state curtails the general form of the strain tensor (20). Only the components of $\Phi$ perpendicular to the fluid columns are relevant, i.e., appear in the strain tensor. Using (39), i.e.,

$$
\begin{aligned}
& \partial_{\alpha} \Phi_{x}=\partial_{\alpha} \Phi_{1}=\left|\nabla \Phi_{1}\right| b_{0 \alpha}, \\
& \partial_{\alpha} \Phi_{y}=\partial_{\alpha} \Phi_{2}=\left|\nabla \Phi_{2}\right| c_{0 \alpha},
\end{aligned}
$$

the strain tensor of a canonic can be written as

$$
U_{\alpha \beta}=\frac{1}{2}\left[\delta_{\alpha \beta}-\left|\nabla \Phi_{1}\right|^{2} b_{0 \alpha} b_{0 \beta}-\left|\nabla \Phi_{2}\right|^{2} c_{0 \alpha} c_{0 \beta}\right] .
$$

Rewriting the strain tensor in terms of $b_{\alpha}$ and $c_{\alpha}$ gives

$$
\begin{aligned}
U_{\alpha \beta}= & \frac{1}{2}\left[\delta_{\alpha \beta}-\frac{1}{2}\left\{\left|\nabla \Phi_{1}\right|^{2}+\left|\nabla \Phi_{2}\right|^{2}+\sqrt{1-\frac{\left(\nabla \Phi_{1} \cdot \nabla \Phi_{2}\right)^{2}}{\left|\nabla \Phi_{1}\right|^{2}\left|\nabla \Phi_{2}\right|^{2}}}\left(\left|\nabla \Phi_{1}\right|^{2}-\left|\nabla \Phi_{2}\right|^{2}\right)\right\} b_{\alpha} b_{\beta}\right. \\
& -\frac{1}{2}\left\{\left|\nabla \Phi_{1}\right|^{2}+\left|\nabla \Phi_{2}\right|^{2}-\sqrt{1-\frac{\left(\nabla \Phi_{1} \cdot \nabla \Phi_{2}\right)^{2}}{\left|\nabla \Phi_{1}\right|^{2}\left|\nabla \Phi_{2}\right|^{2}}}\left(\left|\nabla \Phi_{1}\right|^{2}-\left|\nabla \Phi_{2}\right|^{2}\right)\right\} c_{\alpha} c_{\beta} \\
& \left.-\frac{1}{2} \frac{\nabla \Phi_{1} \cdot \nabla \Phi_{2}}{\left|\nabla \Phi_{1}\right|\left|\nabla \Phi_{2}\right|}\left\{\left|\nabla \Phi_{1}\right|^{2}+\left|\nabla \Phi_{2}\right|^{2}\right\}\left(b_{\alpha} c_{\beta}+c_{\alpha} b_{\beta}\right)\right] .
\end{aligned}
$$


This expression implies immediately that the positional part of the deformation free energy density of a canonic must read,

$$
\begin{aligned}
f_{p}= & \frac{1}{2} \lambda_{1111}\left(b_{\alpha} b_{\beta} U_{\alpha \beta}\right)^{2}+\lambda_{1112}\left(b_{\alpha} b_{\beta} U_{\alpha \beta}\right)\left(b_{\alpha} c_{\beta} U_{\alpha \beta}\right) \\
& +\frac{1}{2} \lambda_{2222}\left(c_{\alpha} c_{\beta} U_{\alpha \beta}\right)^{2}+\lambda_{2212}\left(c_{\alpha} c_{\beta} U_{\alpha \beta}\right)\left(b_{\alpha} c_{\beta} U_{\alpha \beta}\right) \\
& +\frac{1}{2} \lambda_{1212}\left(b_{\alpha} c_{\beta} U_{\alpha \beta}\right)^{2}+\lambda_{1122}\left(b_{\alpha} b_{\beta} U_{\alpha \beta}\right)\left(c_{\alpha} c_{\beta} U_{\alpha \beta}\right),
\end{aligned}
$$

i.e., six elastic constants are required to describe the positional elasticity of a canonic. With the aid of

$$
\begin{aligned}
\varepsilon_{1} & =\frac{1}{2}\left(1-\left|\nabla \Phi_{1}\right|^{2}\right) \\
& =\partial_{x} u_{x}-\frac{1}{2}\left|\nabla u_{x}\right|^{2} \\
\varepsilon_{2} & =\frac{1}{2}\left(1-\left|\nabla \Phi_{2}\right|^{2}\right) \\
& =\partial_{y} u_{y}-\frac{1}{2}\left|\nabla u_{y}\right|^{2} \\
\varepsilon_{3} & =-\frac{1}{2} \nabla \Phi_{1} \cdot \nabla \Phi_{2} \\
& =\frac{1}{2}\left(\partial_{x} u_{y}+\partial_{y} u_{x}\right)-\frac{1}{2} \nabla u_{x} \cdot \nabla u_{y},
\end{aligned}
$$

the three relevant positional invariants can be expressed in terms of $\partial_{\alpha} u_{x}$ and $\partial_{\alpha} u_{y}$ as

$$
\begin{aligned}
b_{\alpha} b_{\beta} U_{\alpha \beta}= & \frac{1}{2}\left(\varepsilon_{1}+\varepsilon_{2}\right) \\
& +\frac{1}{2}\left(\varepsilon_{1}-\varepsilon_{2}\right) \sqrt{1-4 \varepsilon_{3}^{2} /\left(1-2 \varepsilon_{1}\right)\left(1-2 \varepsilon_{2}\right)}, \\
c_{\alpha} c_{\beta} U_{\alpha \beta}= & \frac{1}{2}\left(\varepsilon_{1}+\varepsilon_{2}\right) \\
& -\frac{1}{2}\left(\varepsilon_{1}-\varepsilon_{2}\right) \sqrt{1-4 \varepsilon_{3}^{2} /\left(1-2 \varepsilon_{1}\right)\left(1-2 \varepsilon_{2}\right)}, \\
b_{\alpha} c_{\beta} U_{\alpha \beta}= & \frac{\varepsilon_{3}}{\sqrt{\left(1-2 \varepsilon_{1}\right)\left(1-2 \varepsilon_{2}\right)}}\left(1-\varepsilon_{1}-\varepsilon_{2}\right) .
\end{aligned}
$$

For small deformations, only the terms of order $\varepsilon$ are relevant. This means that the three relevant positional invariants can be replaced by

$$
\begin{aligned}
& b_{\alpha} b_{\beta} U_{\alpha \beta}=\varepsilon_{1}, \\
& c_{\alpha} c_{\beta} U_{\alpha \beta}=\varepsilon_{2}, \\
& b_{\alpha} c_{\beta} U_{\alpha \beta}=\varepsilon_{3} .
\end{aligned}
$$

\section{Orientational elasticity}

Expressions for the nine invariants $D_{i j}$ in terms of the $\partial_{\alpha} \partial_{\beta} u_{x}$ and $\partial_{\alpha} \partial_{\beta} u_{y}$ can be derived from equations (40) and (41). It follows from (40) that

$$
\boldsymbol{a} \cdot(\boldsymbol{\nabla} \times \boldsymbol{a})=0 .
$$

The additional requirement that the canonic columns are incompressible, i.e.,

$$
\begin{array}{r}
\left|\nabla \Phi_{1}\right| \equiv 1 \\
\left|\nabla \Phi_{2}\right| \equiv 1 \\
\nabla \Phi_{1} \cdot \nabla \Phi_{2} \equiv 0
\end{array}
$$

implies that

$$
\begin{aligned}
& \nabla \times b=0, \\
& \nabla \times c=0 .
\end{aligned}
$$

Consequently, the effective description of canonic elasticity only involves the two invariants:

$$
\begin{aligned}
& D_{31}=-\boldsymbol{b} \cdot(\boldsymbol{\nabla} \times \boldsymbol{a}), \\
& D_{32}=-\boldsymbol{c} \cdot(\boldsymbol{\nabla} \times \boldsymbol{a}) .
\end{aligned}
$$

The number of two relevant invariants implies that there are three quadratic first order terms. All three terms are bulk terms, as the surface terms $S_{i j}$ are all zero. The two chiral terms asociated with the two remaining invariants are forbidden, as the state with zero displacement field is, by assumption, the equilibrium state. There are no relevant surface terms of the kind $S_{i j k}$.

\section{DISCUSSION AND CONCLUSION}

The relevant elastic constants for a particular phase are derived from the symmetry requirements:

$$
\begin{aligned}
\lambda_{i j k l} & =T_{i m} T_{j n} T_{k p} T_{l q} \lambda_{m n p q} \\
k_{i j} & =T T_{i m} T_{j n} k_{m n} \\
K_{i j k l} & =T_{i m} T_{j n} T_{k p} T_{l q} K_{m n p q} \\
L_{i j k} & =T T_{i m} T_{j n} T_{k p} L_{m n p},
\end{aligned}
$$

which hold for all elements $T_{i j}$ of the symmetry group of the phase. The quantity $T= \pm 1$ is the determinant of the symmetry transformation $T_{i j}$.

The orientational elastic constants for a general smectic and for the Sm-C (monoclinic $2 / m$ symmetry), Sm$C^{*}, \mathrm{Sm}-C_{\alpha}^{*}, \mathrm{Sm}-C_{\gamma}^{*}$ ( all monoclinic 2-symmetry), Sm$C_{A}^{*}$ (orthorhombic 222-symmetry), and $\mathrm{Sm}-A$ (uniaxial $D_{\infty h}$ symmetry) phases are given in Table I. The result for $\mathrm{Sm}-C$ can be shown to be equivalent to the result of Leslie, Stewart, and Nakagawa [6] and to the result of the Orsay group [7]. However, the treatment of the Orsay group is less general as it holds only for small displacements. Agreement is found with the result of Dahl and Lagerwall [8] and Dahl [9] as well as with the result of Carlsson, Stewart, and Leslie [10] for Sm- $C^{*}$. Agreement is also found with de Gennes in case of small displacements [11]. However, opinions regarding the nature of the equilibrium state differ. Dahl and Lagerwall oppose the assumption that the equilibrium state is the state with spatial periodicity and, therefore, find all chiral terms allowed by symmetry. Carlsson, Stewart, and Leslie only raise the question whether this assumption is justified, whereas de Gennes agrees with the assumption adhered to in the present paper. The elastic constants for Sm- $C^{*}$ given in Table $I$ are related to the elastic constants of the Orsay group [7] and Dahl and Lagerwall [8] according to 
TABLE I. The orientational elastic constants for some relevant smectic mesophases. The linear combinations of elastic constants appear because of $D_{11}+D_{22}=0$. The invariants belonging to the surface elastic constants $K_{i j k l}$ can be expressed as linear combinations of invariants belonging to the bulk elastic constants $K_{i j k l}$ and pure surface terms.

\begin{tabular}{|c|c|c|c|c|}
\hline Smectic phase & $\begin{array}{c}k_{i j} \\
\text { bulk }\end{array}$ & $K_{i j k l}$ & surface & $\begin{array}{c}L_{i j k} \\
\text { surface }\end{array}$ \\
\hline General & $k_{33}$ & $\begin{array}{c}K_{1111}+K_{2222}-2 K_{1122} \\
K_{1112}-K_{2212}, K_{1121}-K_{2221} \\
K_{1123}-K_{2223}, K_{1113}-K_{2213} \\
K_{1212}, K_{2121}, K_{3333}, K_{2323} \\
K_{3321}, K_{2333}, K_{2321}, K_{1333} \\
K_{1312}, K_{1313}, K_{1323}\end{array}$ & $\begin{array}{c}K_{1133}-K_{2233} \\
K_{3312}, K_{1221} \\
K_{2312}, K_{1321}\end{array}$ & $L_{333}$ \\
\hline $\mathrm{Sm}-C$ & & $\begin{array}{c}K_{1111}+K_{2222}-2 K_{1122} \\
K_{1123}-K_{2223}, K_{1212} \\
K_{2121}, K_{3333}, K_{2323}, K_{2333} \\
K_{1313}, K_{1312}\end{array}$ & $\begin{array}{c}K_{1133}-K_{2233} \\
K_{1221}, K_{1321}\end{array}$ & \\
\hline $\begin{array}{l}\mathrm{Sm}-C^{*}, \mathrm{Sm}-C_{\alpha}^{*} \\
\mathrm{Sm}-C_{\gamma}^{*}\end{array}$ & $k_{33}$ & $\begin{array}{c}K_{1111}+K_{2222}-2 K_{1122} \\
K_{1123}-K_{2223}, K_{1212} \\
K_{2121}, K_{3333}, K_{2323}, K_{2333} \\
K_{1313}, K_{1312}\end{array}$ & $\begin{array}{c}K_{1133}-K_{2233} \\
K_{1221}, K_{1321}\end{array}$ & \\
\hline $\mathrm{Sm}-C_{\boldsymbol{A}}^{*}$ & $k_{33}$ & $\begin{array}{c}K_{1111}+K_{2222}-2 K_{1122} \\
K_{1212}, K_{2121}, K_{3333} \\
K_{2323}, K_{1313}\end{array}$ & $\begin{array}{c}K_{1133}-K_{2233} \\
K_{1221}\end{array}$ & \\
\hline $\mathrm{Sm}-A$ & & $\begin{array}{c}K_{1212}=K_{2121}= \\
-K_{1122}-K_{1221}+\frac{1}{2}\left(K_{1111}+K_{2222}\right)\end{array}$ & $K_{1221}$ & \\
\hline
\end{tabular}

$$
\begin{aligned}
D & =-k_{33} \\
A_{11} & =K_{1111}+K_{2222}-2 K_{1122}-2 K_{1221} \\
A_{12} & =K_{2121} \\
A_{21} & =K_{1212} \\
B_{1} & =K_{2323} \\
B_{2} & =K_{1313} \\
B_{3} & =K_{3333} \\
B_{13} & =K_{2333} \\
C_{1} & =K_{1123}-K_{2223}+K_{1321} \\
C_{2} & =K_{1312} \\
E_{5} & =K_{1221} \\
E_{6} & =-K_{1133}+K_{2233} \\
E_{7} & =K_{1321}
\end{aligned}
$$

Results are presented for the antiferroelectric $\mathrm{Sm}-C_{A}^{*}$ [12], and for the ferrielectric $\mathrm{Sm}-C_{\alpha}^{*}$ and $\mathrm{Sm}-C_{\gamma}^{*}[13]$ phases. It should be noted that the macroscopic symmetry of the ferrielectric chiral smectics is the same as that of the ferroelectric $\mathrm{Sm}-C^{*}$, although their microscopic structures differ. This means that, as far as the form of the deformation free energy density is concerned, the ferrielectric and ferroelectric chiral smectics do not differ.

The relevant positional and orientational elastic constants for a general canonic and for the tilted canonic $D_{t}$ (monoclinic $2 / m$ symmetry with $b$ as symmetry axis), the rectangular canonic $D_{r d}$ (glide plane perpendicular to $\boldsymbol{b}$, i.e., the point group symmetry is monoclinic), and the hexagonal canonic $D_{h d}(6 / \mathrm{mmm}$ symmetry with $a$ as sixfold axis) are given in Table II. The subscript $d$ indicates that the molecular organization in the columns is disordered, i.e., fluidlike. See, for the full nomenclature of these phases, Ref. [14]. Agreement is found with the hydrodynamic theory of Brand and Pleiner [15] in the cases of monoclinic and hexagonal canonics, and with the theory of Prost and Clark [16] in the case of hexagonal canonics.

Summarizing, a formulation of the theory of elasticity of smectic and canonic mesophases is presented in terms of a fully covariant notation. The theory is founded on the interdependence of the positional and orientational deformation variables, as required by the macroscopic definition of these phases. The deformation free energy density consists of two independent contributions, namely, the contributions due to the positional deformations and to the orientational deformations. The general expression of the deformation free energy density of an incompressible nonchiral smectic involves 1 positional elastic constant, 16 bulk orientational elastic constants and 6 surface orientational elastic constants, whereas the corresponding expression for an incompressible non-chiral canonic involves six positional elastic constants and three bulk orientational elastic constants. Chirality involves only one additional bulk orientational elastic constant for smectics, but none for canonics. 
TABLE II. The positional and orientational elastic constants for some relevant canonic mesophases.

\begin{tabular}{lcc}
\hline \hline Canonic phase & $\lambda_{i j k l}$ & $K_{i j k l}$ \\
\hline General & $\lambda_{1111}, \lambda_{2222}, \lambda_{1122}, \lambda_{1212}, \lambda_{1112}, \lambda_{2212}$ & $K_{3131}, K_{3232}, K_{3132}$ \\
$D_{\mathrm{t}}$ & $\lambda_{1111}, \lambda_{2222}, \lambda_{1122}, \lambda_{1212}$ & $K_{3131}, K_{3232}$ \\
$D_{\text {rd }}$ & $\lambda_{1111}, \lambda_{2222}, \lambda_{1122}, \lambda_{1212}$ & $K_{3131}, K_{3232}$ \\
$D_{\text {hd }}$ & $\lambda_{1212}, \lambda_{1111}=\lambda_{2222}=\lambda_{1122}+\frac{1}{2} \lambda_{1212}$ & $K_{3131}=K_{3232}$ \\
\hline \hline
\end{tabular}

\section{APPENDIX}

For the sake of simplicity the strain tensor is approximated by

$$
U_{\alpha \beta}=\frac{1}{2}\left(\partial_{\alpha} u_{\beta}+\partial_{\alpha} u_{\beta}\right) .
$$

The most general surface term that can be constructed as a linear combination of terms quadratic in $U_{\alpha \beta}$ reads

$$
\partial_{\alpha}\left(A_{\alpha \beta \gamma \delta} u_{\beta} \partial_{\gamma} u_{\delta}\right)
$$

where the tensor $A_{\alpha \beta \gamma \delta}$ is composed of the three local axes $\boldsymbol{a}, \boldsymbol{b}$, and $\boldsymbol{c}$. This tensor is completely determined by the following two properties. First, the surface term must be solely a linear combination of terms quadratic in $\partial_{\alpha} u_{\beta}$. This means that the surface term does not contain terms linear in $u_{\alpha}$, nor terms linear in $\partial_{\alpha} \partial_{\beta} u_{\gamma}$. With the aid of

$$
\begin{aligned}
\partial_{\alpha}\left(A_{\alpha \beta \gamma \delta} u_{\beta} \partial_{\gamma} u_{\delta}\right)= & \left(\partial_{\alpha} A_{\alpha \beta \gamma \delta}\right) u_{\beta} \partial_{\gamma} u_{\delta}+A_{\alpha \beta \gamma \delta} u_{\beta} \partial_{\alpha} \partial_{\gamma} u_{\delta} \\
& +A_{\alpha \beta \gamma \delta} \partial_{\alpha} u_{\beta} \partial_{\gamma} u_{\delta}
\end{aligned}
$$

it can be directly concluded that

$$
\begin{aligned}
\partial_{\alpha} A_{\alpha \beta \gamma \delta} & =0, \\
A_{\alpha \beta \gamma \delta} & =-A_{\gamma \beta \alpha \delta} .
\end{aligned}
$$

Second, the surface term must be a linear combination of terms quadratic in the strain tensor $U_{\alpha \beta}$. Consequently, the tensor $A_{\alpha \beta \gamma \delta}$ satisfies the symmetry relations:

$$
\begin{aligned}
& A_{\alpha \beta \gamma \delta}=A_{\beta \alpha \gamma \delta}, \\
& A_{\alpha \beta \gamma \delta}=A_{\alpha \beta \delta \gamma} .
\end{aligned}
$$

The relation (A1a) implies that the tensor $A_{\alpha \beta \gamma \delta}$ must be constructed of the constant Kronecker tensor $\delta_{\alpha \beta}$ alone, i.e., $A_{\alpha \beta \gamma \delta}$ can be written as

$$
A_{\alpha \beta \gamma \delta}=c_{1} \delta_{\alpha \beta} \delta_{\gamma \delta}+c_{2} \delta_{\alpha \gamma} \delta_{\beta \delta}+c_{3} \delta_{\alpha \delta} \delta_{\beta \gamma}
$$

where $c_{1}, c_{2}$, and $c_{3}$ are arbitrary constants. According to the relation $(\mathrm{A} 1 \mathrm{~b})$, the constants $c_{1}, c_{2}$, and $c_{3}$ must satisfy the equations:

$$
\begin{aligned}
& c_{1}=-c_{3}, \\
& c_{2}=0 .
\end{aligned}
$$

The relations (A2) lead to the equation

$$
c_{2}=c_{3}
$$

Clearly, it holds that $c_{1}=c_{2}=c_{3}=0$, i.e.,

$$
A_{\alpha \beta \gamma \delta}=0 \text {, }
$$

meaning that there are no surface terms present in the deformation free energy density due to positional elasticity.
[1] L.D. Landau and E.M. Lifshitz, The Theory of Elasticity (Pergamon Press, Oxford, 1986).

[2] S. Stallinga and G. Vertogen, Phys. Rev. E 49, 1483 (1994).

[3] M. Kleman and O. Parodi, J. Phys. (Paris) 36, 671 (1975).

[4] R. Holyst and A. Poniewierski, J. Phys. II (France) 3, 177 (1993).

[5] C.W. Oseen, Trans. Faraday Soc. 29, 883 (1933).

[6] F.M. Leslie, I.W. Stewart, and M. Nakagawa, Mol. Cryst. Liq. Cryst. 198, 443 (1990).

[7] Orsay Liquid Crystal Group, Solid State Commun. 9, 653 (1971).

[8] I. Dahl and S. T. Lagerwall, Ferroelectrics 58, 215 (1984).

[9] I. Dahl, Ferroelectrics 113, 103 (1991); 113, 121 (1991).

[10] T. Carlsson, I.W. Stewart, and F.M. Leslie, J. Phys. A
25, 2371 (1992).

[11] P.G. de Gennes, The Physics of Liquid Crystals (Clarendon Press, Oxford, 1974).

[12] A.D.L. Chandani, Y. Ouchi, H. Takezoe, A. Fukuda, K. Terashima, and A. Kishi, Jpn. J. Appl. Phys. 28, L1261 (1989); A.D.L. Chandani, E. Gorecka, Y. Ouchi, H. Takezoe, and A. Fukuda, ibid. 28, L1265 (1989).

[13] H. Takezoe, J. Lee, Y. Ouchi, and A. Fukuda, Mol. Cryst. Liq. Cryst. 202, 85 (1991).

[14] W.H. de Jeu and G. Vertogen, Thermotropic Liquid Crystals, Fundamentals, edited by F. P. Schäfer, Springer Series in Chemical Physics Vol. 45 (Springer-Verlag, Berlin, 1988).

[15] H. Brand and H. Pleiner, Phys. Rev. A 24, 2777 (1981).

[16] J. Prost and N.A. Clark, in Liquid Crystals, edited by S. Chandrasekhar (Wiley, New York, 1980). 